\title{
Excitonic Effect Drives Ultrafast Dynamics in Van der Waals Heterostructures Supporting Information
}

\author{
Junyi liuं, Xu Zhang ${ }^{\dagger}$, and Gang Lu* ${ }^{*} \dagger$ \\ tDepartment of Physics and Astronomy, California State University, Northridge,
}

Northridge, California 91330-8268, United States

Section $\mathrm{S} 1 . \mathrm{MoS}_{2} / \mathrm{WS}_{2}$ heterostructure in different stacking configurations

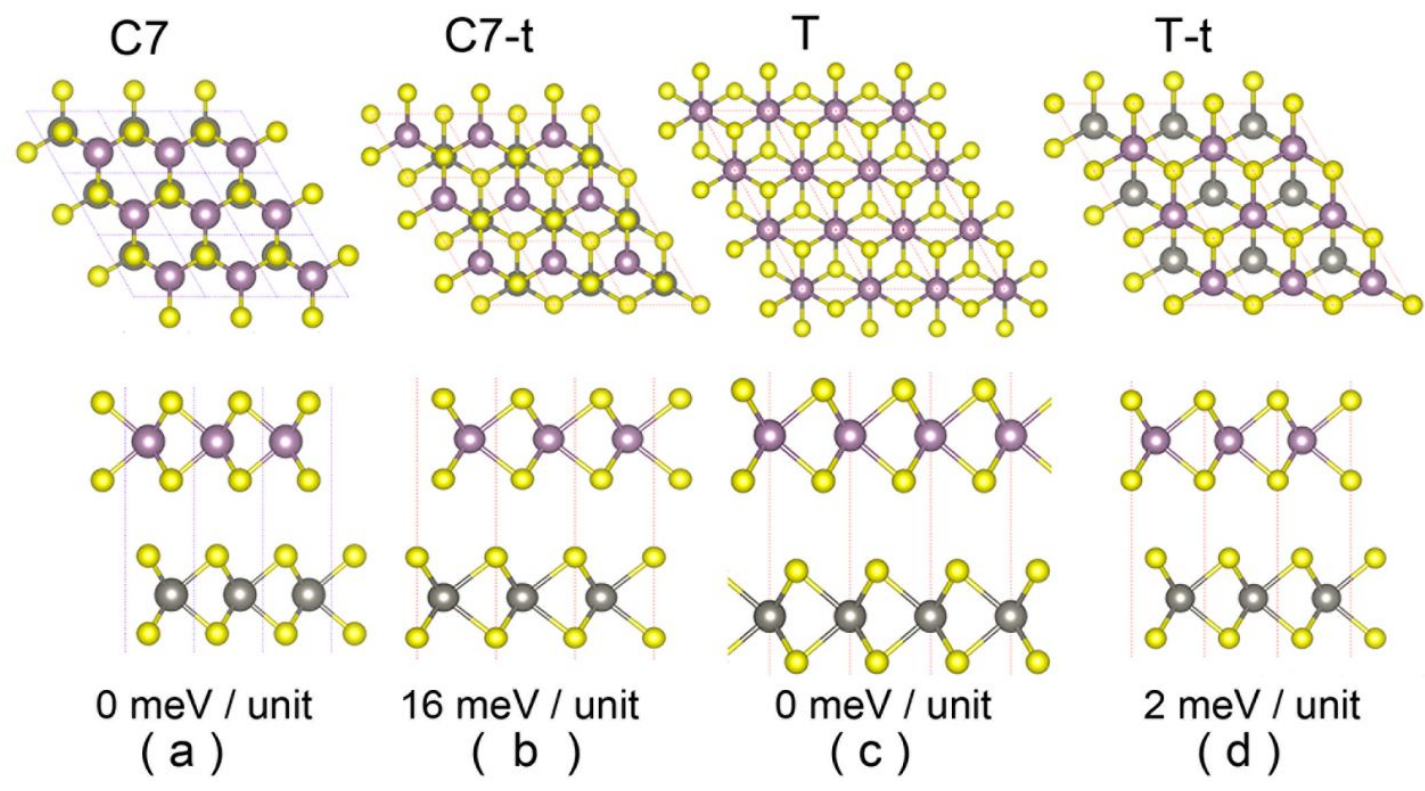

Figure S1. The top (up) and side (bottom) view of (a) C7, (b) C7-t, (c) T and (c) T-t stacking configuration. The total energy per unit cell relative to that of $\mathrm{C} 7$ is listed at the bottom.

\section{Section S2. Computational methods}

Determining exciton states. To determine the energies and many-body wavefunctions of the excitons, we employ the linear-response time-dependent density functional theory (LR-TDDFT) $)^{1,2}$ with an optimally tuned, screened and rangeseparated hybrid exchange-correlation functionals (OT-SRSH) ${ }^{3}$. The OT-SRSH 
involves the partition of the Coulombic interaction into a short-range and a long-range contribution based on the following expression ${ }^{4}$

$$
\frac{1}{r}=\frac{1-[\alpha+\beta \operatorname{erf}(\gamma r)]}{r}+\frac{\alpha+\beta \operatorname{erf}(\gamma r)}{r} .
$$

The hybrid exchange-correlation (XC) functional can be expressed as

$$
E_{x c}^{R S H}=\alpha E_{x x}+\beta E_{x x}^{L R}+(1-\alpha) E_{K S x}-\beta E_{K S x}^{L R}+E_{K S c} .
$$

Where $E_{x x} / E_{x x}^{L R}$ is the Fock-like exact exchange energy, and $E_{K S x} / E_{K S x}^{L R}$ and $E_{K S c}$ are the semilocal Kohn-Sham (KS) exchange and correlation energy, respectively. LR labels the long-range $\mathrm{XC}$ terms. $\alpha$ determines the contribution from the exact exchange and $\beta$ controls the contribution from the long-range exchange terms. $\gamma$ is the range-separation parameter. Additionally, $\alpha$ and $\beta$ satisfy the requirement of $\alpha+\beta=\varepsilon_{0}{ }^{-1}$, where $\varepsilon_{0}$ is the scalar dielectric constant of the solid, thereby enforcing the correct asymptotic screening of the Coulomb tail ${ }^{5}$. The OT-SRSH functional is able to reproduce the correct long-range electron-electron and electron-hole interactions in solids by choosing the reasonable parameters. In order to reduce the computational cost associated with the Fock-like exchange on large systems, we apply first-order perturbation theory to the range-separated hybrid Kohn-Sham (RSH-KS) Hamiltonian and obtain the first-order corrected RSH-KS eigenvalues and eigenfunctions ${ }^{6,7}$. We then solve the following non-Hermitian eigenvalue equations of $\mathrm{Casida}^{8}$,

$$
\left(\begin{array}{cc}
A & B \\
B^{*} & A^{*}
\end{array}\right)\left(\begin{array}{c}
X_{I} \\
Y_{I}
\end{array}\right)=\omega_{I}\left(\begin{array}{cc}
1 & 0 \\
0 & -1
\end{array}\right)\left(\begin{array}{c}
X_{I} \\
Y_{I}
\end{array}\right)
$$

where the pseudo-eigenvalue $\omega_{\mathrm{I}}$ is the I-th exciton energy level. The matrix elements of $\mathbf{A}$ and $\mathbf{B}$ in the basis of KS states ( $i j \sigma)$ are given by

$$
\begin{gathered}
A_{i j \sigma, k l \tau}=\delta_{i, k} \delta_{j, l} \delta_{\sigma, \tau}\left(\varepsilon_{j \sigma}-\varepsilon_{i \sigma}\right)+K_{i j \sigma, k l \tau} \\
B_{i j \sigma, k l \tau}=K_{i j \sigma, l k \tau}
\end{gathered}
$$


Here $K$ is the coupling matrix where indices $i$ and $k$ indicate the occupied orbitals, and $j$ and $l$ represent the virtual KS orbitals. According to the assignment ansatz of Casida, the many-body wavefunction of an excited state $I$ can be written as

$$
\Phi_{I} \approx \sum_{i j \sigma} \frac{X_{I, i j \sigma}+Y_{I, i j \sigma}}{\sqrt{\omega_{I}}} \hat{a}_{j \sigma}^{\dagger} \hat{a}_{i \sigma} \Phi_{0}=\sum_{i j \sigma} Z_{I, i j} \hat{a}_{j \sigma}^{\dagger} \hat{a}_{i \sigma} \Phi_{0}
$$

where $z_{I, i j}=\left(\boldsymbol{X}_{I, i j}+\boldsymbol{Y}_{I, i j}\right) / \sqrt{\omega_{I}} ; \hat{a}_{i \sigma}$ is the annihilation operator acting on the $i$ th $\mathrm{KS}$ orbital with spin $\sigma$ and $\Phi_{0}$ is the ground-state many-body wave function taken to be the single Slater determinate (SD) of the occupied KS orbitals.

Exciton transition rates. The thermal fluctuations of the ions could lead to an overlap between the exciton states in space and energy, and thus promote transitions between the excitonic states. Since these transitions can be nonadiabatic, the nonadiabatic ab initio molecular dynamics is used to describe the phonon-assisted exciton transitions ${ }^{9}$. During a molecular dynamics trajectory, the time-dependent manybody wave function of the excitonic state $\Psi(\mathrm{t})$ is expanded by a linear combination of a complete basis set consisting of the adiabatic ground state and the excited states $\Phi_{I}$ $[\mathbf{R}(\mathrm{t})]$ at the present ionic positions $\mathbf{R}(\mathrm{t})$ :

$$
\Psi(t)=\sum_{I=0}^{\infty} C_{I}(t) \Phi_{I}[R(t)]
$$

where $C_{I}(t)$ is the expansion coefficient. Let the exciton start in a pure state $I$ at $t=0$ (i.e., $\left.\Phi_{I}[\mathbf{R}(\mathrm{t})]\right)$ then the coefficient $C_{J}(t)$ in Eq. (7) can be labeled as $C_{f}^{(I)}(t)$ with the initial condition that $C \int^{(I)}(0)=\delta_{I, J}$. At $\mathrm{t}>0$, ions move and $\Psi(\mathrm{t})$ becomes a mixed state. Therefore $\mid C\}\left.^{I)}(t)\right|^{2}$ represents the probability that the exciton makes a transition from state $I$ to state $J$ during a small time interval of $t$.

The evolution of $C_{J}(t)$ can be determined from the nonadiabatic molecular dynamics (NAMD) at each time step. Substituting Eq. (7) into the time-dependent Schrödinger equation, one arrives at the following equation involving the expansion coefficient $C_{J}(t)$ : 


$$
\frac{\partial}{\partial t} C_{J}(t)=-\sum_{K} C_{K}(t)\left(\frac{i}{\mathrm{~h}} \omega_{K} \delta_{J K}+D_{J K}\right)(8)
$$

A standard second-order finite-difference method with a timestep of $10^{-3} \mathrm{fs}$ is employed to propagate the coefficient $C_{J}(t)$. And $D_{J K}$ is the nonadiabatic coupling between two many-body electronic states $J$ and $K$,

$$
D_{J K} \equiv\left\langle\Phi_{J}\left|\nabla_{R}\right| \Phi_{K}\right\rangle \cdot \frac{d R}{d t}=\left\langle\Phi_{J}\left|\frac{\partial}{\partial t}\right| \Phi_{K}\right\rangle
$$

Substituting Eq. (6) into Eq. (9), we can obtain $D_{J K}$ as

$$
D_{J K}=\sum_{i i i^{\prime}, j j^{\prime}} Z_{J, i j}^{*} Z_{K, i^{\prime} j^{\prime}}\left\langle\Phi_{i j}\left|\frac{\partial}{\partial t}\right| \Phi_{i^{\prime} j^{\prime}}\right\rangle=\sum_{i, j \neq j^{\prime}} Z_{J, i j}^{*} Z_{K, i^{\prime} j^{\prime}} d_{j j^{\prime}}-\sum_{i \neq i^{\prime}, j} Z_{J, i j}^{*} Z_{K, i^{\prime} j^{\prime}} d_{i i^{\prime}}
$$

where $\Phi_{i j}=\hat{a}_{j}^{\dagger} \hat{a}_{i} \Phi_{0}$ is a single SD of the KS orbitals, promoting an electron from an occupied state $\mathrm{i}$ to a virtual state $\mathrm{j} . \mathrm{d}_{j k}=\left\langle\phi_{j}\left|\frac{\partial}{\partial t}\right| \phi_{k}\right\rangle$ is the nonadiabatic coupling between the KS orbitals. The nonadiabatic coupling between the exciton state $J$ and the ground state is obtained as

$$
D_{J 0}=\sum_{i, j} Z_{J, i j}^{*} d_{j i}
$$

Hole transition rates without excitonic effect. Here, we only consider the evolution of a single quasi-particle. During a molecular dynamic trajectory, the timedependent single-particle wavefunction $\varphi(t)$ can be expanded by a linear combination of a complete basis set consisting of the time-dependent KS orbitals $(\phi(\mathbf{R}(\mathrm{t}))$ at the present ionic positions $\mathbf{R}(\mathrm{t})$.

$$
\varphi(t)=\sum_{i} c_{i}(t) \phi_{i}(R(t))
$$

Starting from a pure hole (or electron) state $\left(\varphi(0)=\phi_{i}(\mathrm{R}(0))\right.$, the coefficients can be labeled as $c_{j}^{(i)}(\mathrm{t}) \quad$ with initial condition $c_{j}^{i}(0)=\delta_{i j}$. At $\mathrm{t}>0$, the pure state becomes a mixed state, and thus $\left|c_{j}^{(i)}(t)\right|^{2}$ represents the probability that the hole (or electron) makes a transition from state $\mathrm{i}$ to $\mathrm{j}$. The evolution of the single-particle wavefunction 
is determined by the time-dependent KS equation. Then we can obtain equations for the expanding coefficients

$$
\frac{\partial}{\partial t} c_{j}(t)=-\sum_{k} \frac{i}{\mathrm{~h}} c_{k}(t)\left(\varepsilon_{k} \delta_{j k}+d_{j k}\right)
$$

Where $\mathrm{d}_{j k}=\left\langle\phi_{j}\left|\frac{\partial}{\partial t}\right| \phi_{k}\right\rangle$ is the nonadiabatic coupling between the KS orbitals.

Table S1. The calculated fundamental gap $\left(\mathrm{E}_{\mathrm{g}}\right)$, optical gap $\left(\mathrm{E}_{\mathrm{opt}}\right)$ and exciton binding energy $\left(\mathrm{E}_{\mathrm{b}}\right)$ for $\mathrm{MoS}_{2}, \mathrm{WS}_{2}$ monolayer and $\mathrm{MoS}_{2} / \mathrm{WS}_{2}$ heterostructure using different methods of (TD)DFT-OT-SRSH and GW-BSE, respectively. All energies are in $\mathrm{eV}$.

(TD)DFT-OT-SRSH

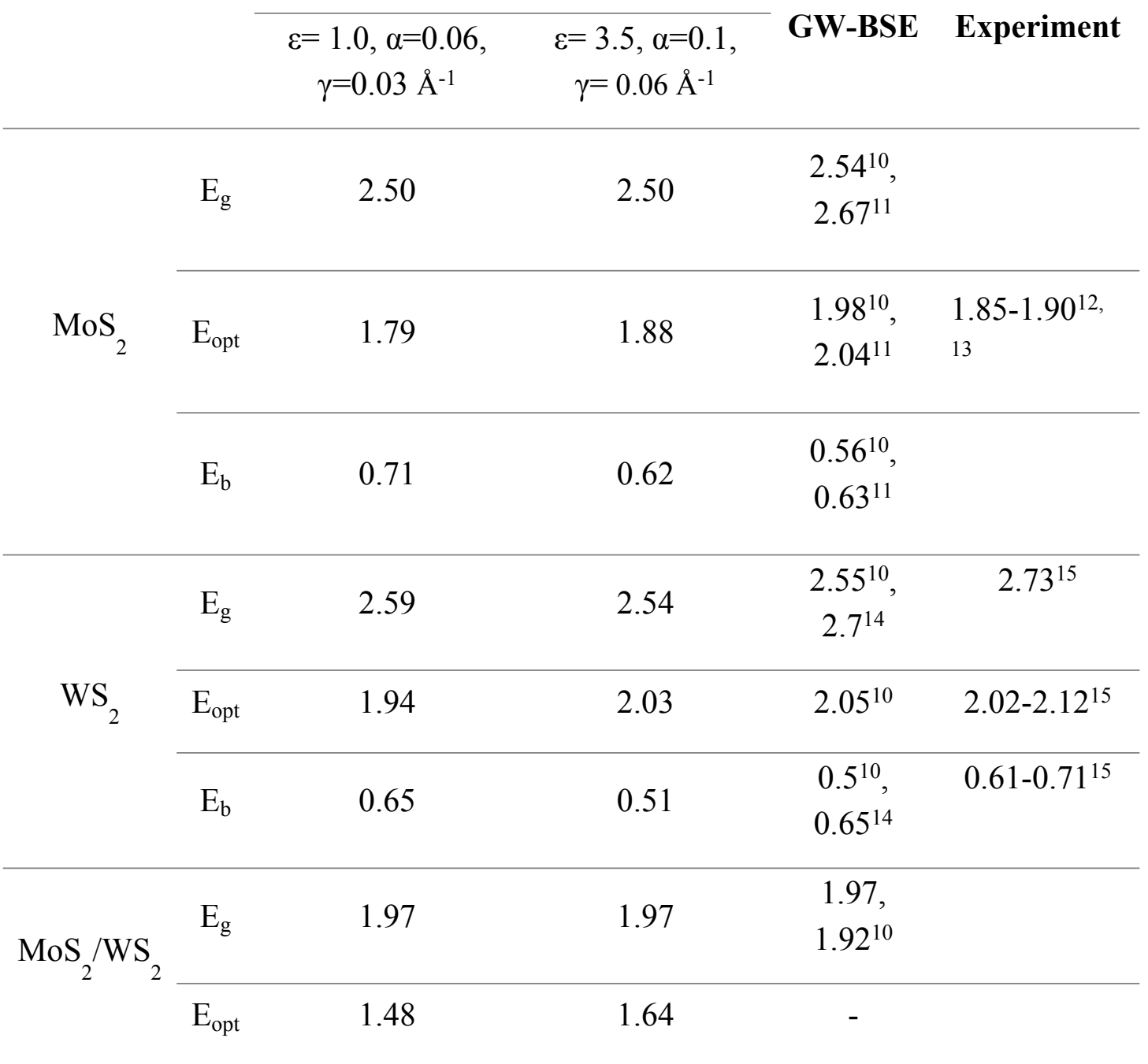



$\mathrm{E}_{\mathrm{b}}$
0.49
0.33

Table S2. The calculated energy $(\mathrm{eV})$ of $\mathrm{S}_{\mathrm{I}}, \mathrm{S}_{\mathrm{M} 1}, \mathrm{~S}_{\mathrm{M} 2}, \mathrm{~S}_{\mathrm{M} 2}$, and $\mathrm{S}_{0}$ for $\mathrm{MoS}_{2} / \mathrm{WS}_{2}$ heterostructure with different parameters. $\Delta \mathrm{S}$ is the minimal energy difference between $\mathrm{S}_{\mathrm{I}}$ and $\mathrm{S}_{\mathrm{M} 1} / \mathrm{S}_{\mathrm{M} 2} / \mathrm{S}_{\mathrm{M} 3}$.

\begin{tabular}{|c|c|c|c|c|c|c|}
\hline Parameters & $\mathrm{S}_{0}$ & $\mathrm{~S}_{\mathrm{I}}$ & $\mathrm{S}_{\mathrm{M} 1}$ & $\mathrm{~S}_{\mathrm{M} 2}$ & $\mathrm{~S}_{\mathrm{M} 3}$ & $\Delta \mathbf{S}$ \\
\hline$\varepsilon=1.0, \alpha=0.06, \gamma=0.03 \AA^{-1}$ & 1.48 & 1.83 & 1.85 & 1.74 & 1.80 & 0.02 \\
\hline$\varepsilon=3.5, \alpha=0.1, \gamma=0.06 \AA^{-1}$ & 1.64 & 1.89 & 1.93 & 1.98 & 1.95 & 0.04 \\
\hline
\end{tabular}

\section{Section S3. Parameters test of OT-SRSH functional}

For (TD)DFT-OT-SRSH calculations, the scalar dielectric constant $\varepsilon_{0}$ is set as 1.0, which is the correct asymptotic limit for screening in $2 \mathrm{D}$ materials ${ }^{16}$. The other two independent parameters $\alpha=0.06$ and $\gamma=0.03$ are determined by reproducing the fundamental gap of $\mathrm{MoS}_{2} / \mathrm{WS}_{2}$ heterojunction (1.97 eV) obtained from the one-shot GW calculations without spin-orbit coupling (SOC). The experimental and GW-BSE values with SOC are listed in Table S1 for reference. Although the method of choice here is within the most advanced DFT frameworks and can indeed describe excitonic states very well, but it is still limited in the form of the interaction potential and a small number of parameters. To check how the various energies could change with a different set of parameters of OT-SRSH functional, we include in Table S1 the gaps and exciton binding energies with two different sets of parameters. In particular, a much larger dielectric constant $(\varepsilon=3.5)$ is used in one of them. As expected, as the dielectric constant increases, the exciton binding energies decrease. However, as shown in Table S2, the energies of all relevant excitons increase simultaneously as $\varepsilon$ increases from 1 to 3.5 . As a result, the minimal energy difference between the intralayer exciton $\mathrm{S}_{\mathrm{I}}$ and the interlayer exciton $\mathrm{S}_{\mathrm{M} 1} / \mathrm{S}_{\mathrm{M} 2} / \mathrm{S}_{\mathrm{M} 3}$ remains small $(\sim 0.04 \mathrm{eV})$. Thus, our conclusion is unchanged. 


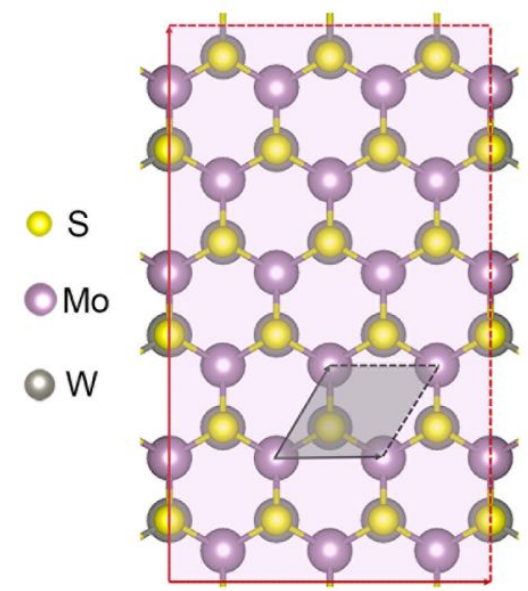

( a )

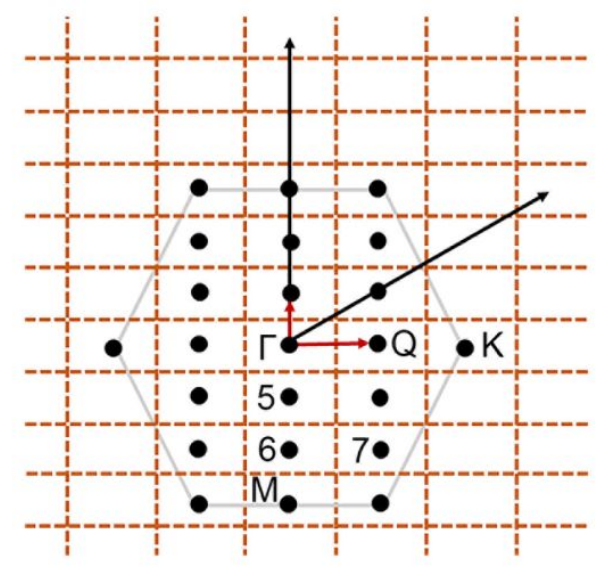

(b)

Figure S2. (a) The structure of the orthogonal $(3 \times 3)$ supercell and the primitive unit cell of $\mathrm{MoS}_{2} / \mathrm{WS}_{2}$ in $\mathrm{C} 7$ stacking. (b) The first Brillouin zone (BZ) of the hexagonal primitive unit cell and the orthogonal $(3 \times 3)$ supercell is represented by the gray hexagon and brown dashed rectangle, respectively. The special $k$-points in the BZ are labeled.

\section{Section S4. Band unfolding}

The excitonic states under study here are many-body states, which are linear combinations of single-particle excitations at different $k$-points (in terms of the Slater determinants). More specifically, we have calculated the excited states in a $(3 \times 3)$ supercell with eight highest occupied Kohn-Sham (KS) orbitals and six lowest unoccupied KS orbitals sampled at the $\Gamma$ point. As shown in Figure 1(b), the first Brillouin zone (BZ) of the hexagonal primitive unit cell is represented by the large gray hexagon while the first $\mathrm{BZ}$ of the orthogonal $(3 \times 3)$ supercell is represented by the small, dashed brown rectangle. The black dots are the $\Gamma$ point and its reciprocal translations for the $(3 \times 3)$ supercell. By comparing the BZs of the $(3 \times 3)$ supercell and the primitive unit cell, we find that seven special $k$-points, including $\Gamma, \mathrm{K}, \mathrm{M}$ and $\mathrm{Q}$ of the primitive unit cell can be folded exactly into the $\Gamma$ point of the $(3 \times 3)$ supercell (i.e., 
they coincide with $\Gamma$ ). By further comparing the energies of the KS orbitals obtained in the $(3 \times 3)$ supercell at the $\Gamma$ point with those in the primitive unit cell at the special $k$ points, we can find the correspondence between the KS orbitals at the $\Gamma$ point of the supercell and those at the special $k$-points of the primitive cell. The indirect band gap between the $\Gamma$ and $\mathrm{K}$ points in the primitive cell is also recovered by the direct band gap at the $\Gamma$ point of the supercell.

The eight highest occupied KS orbitals and six lowest unoccupied KS orbitals form 48 single-particle excitations, which are the basis sets to expand the many-body excitonic wavefunctions. By solving the eigenvalue equations of Casida, we can determine the expansion coefficients. Although the excitonic wavefunctions are superpositions of 48 single-particle excitations, we can identify one single-particle excitation that dominates the excitonic state. Since we know the origins of all KS orbitals in terms of $k$-points, we can relate each excitonic state in the supercell to the dominant single-particle excitations in the primitive cell. During the NAMD simulations, the expansion coefficients change as time. And from the coefficients, we can identify the dominant single-particle excitation (between different $k$-points) that gives rise to the excitonic transition. In other words, the excitations between different $k$-points in the primitive unit cell is captured by the excitations at the $\Gamma$ point of the supercell.

Since our calculations are carried out in the $(3 \times 3)$ supercell at the $\Gamma$ point, there is no change to the total momentum of the excitons. However, each single-particle excitation that constitutes the excitonic state could have momentum exchange between them, but their net sum is conserved.

To verify that 48 excitons in a $(3 \times 3)$ supercell are sufficient to capture exciton dynamics of the system, we also include 18 highest occupied (KS) orbitals and 18 lowest unoccupied KS orbitals in the calculations to examine 324 excitons. The energy and charge density of the low-lying excited states are the same as those of 48 excitons, suggesting convergent results. 


\section{Section S5. Time evolution of the exciton states at $300 \mathrm{~K}$}

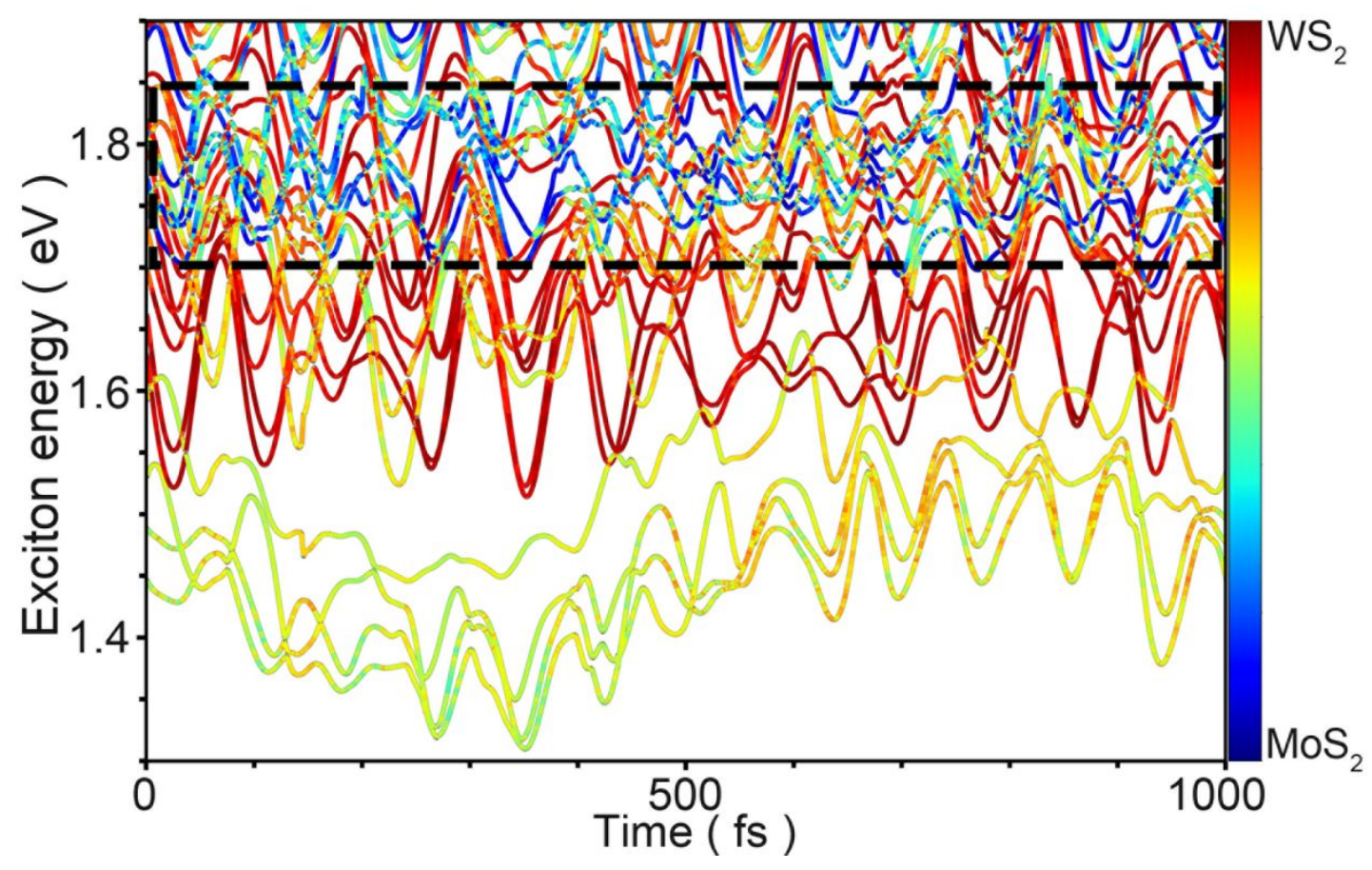

Figure S3. Time evolution of the exciton states in C7 stacking configuration at $300 \mathrm{~K}$. The dashed black box highlights the energy range of initial intralayer excitons $\left(\mathrm{S}_{\mathrm{I}}\right)$. The projection of the hole density onto $\mathrm{WS}_{2}$ (deep red) and $\mathrm{MoS}_{2}$ (deep blue) for each exciton is color-coded according to the side bar.

\section{Section S6. Spin-orbit coupling (SOC) effect}

In order to estimate the SOC effect on the exciton dynamics, we calculate the band structures with and without SOC on the basis of HSE06 functionals. The results are presented in the Figure S4. We find that SOC has little effect on the orbital hybridization and SOC splitting mainly takes place between the valence bands at $\mathrm{K}$ valley. For the valence bands at $\mathrm{WS}_{2} @ \mathrm{~K}$ and $\mathrm{MoS}_{2} @ \mathrm{~K}$, one doubly-occupied band splits into two singly-occupied ones with SOC included, one upshifted and the other downshifted. The values for the SOC splitting at $\mathrm{WS}_{2} @ \mathrm{~K}$ and $\mathrm{MoS}_{2} @ \mathrm{~K}$ are $0.44 \mathrm{eV}$ and $0.19 \mathrm{eV}$, respectively. Considering that the experimentally observed A exciton in $\mathrm{MoS}_{2}$ monolayer comprises the upshifted bands, the band gap should decrease in the 
presence of SOC effect, thus energy separation between the excitonic states should be even smaller. We calculate the single-particle energies of $\mathrm{E}_{\mathrm{I}}\left(\mathrm{MoS}_{2} @ \mathrm{~K}-\mathrm{MoS}_{2} @ \mathrm{~K}\right)$ and E $\mathrm{E}_{\mathrm{M} 1}\left(\mathrm{WS}_{2} @ \mathrm{~K}-\mathrm{MoS}_{2} @ \mathrm{Q}\right) / \mathrm{E}_{\mathrm{M} 2}\left(\mathrm{WS}_{2} @ \mathrm{~K}-\mathrm{MoS}_{2} @ \mathrm{~K}\right) / \mathrm{E}_{\mathrm{M} 3} \quad\left(\mathrm{WS}_{2} @-\mathrm{WS}_{2} @ \mathrm{~K}\right), \quad$ which correspond to the excitons $\mathrm{S}_{\mathrm{I}}$ and $\mathrm{S}_{\mathrm{M} 1} / \mathrm{S}_{\mathrm{M} 2} / \mathrm{S}_{\mathrm{M} 3}$, respectively. As shown in the Table $\mathrm{S} 2$, the absolute value of the minimal energy difference $\Delta\left(E_{I M}\right)$ between $E_{I}$ and $\mathrm{E}_{\mathrm{M} 1} / \mathrm{E}_{\mathrm{M} 2} / \mathrm{E}_{\mathrm{M} 3}$ remains very small with SOC effect considered, suggesting strong energy overlaps between $\mathrm{S}_{\mathrm{I}}$ and $\mathrm{S}_{\mathrm{M} 1} / \mathrm{S}_{\mathrm{M} 2} / \mathrm{S}_{\mathrm{M} 3}$. Therefore, the inclusion of SOC further reduces the energy gaps for ultrafast charge transfer and thus upholds the main conclusions of the paper.

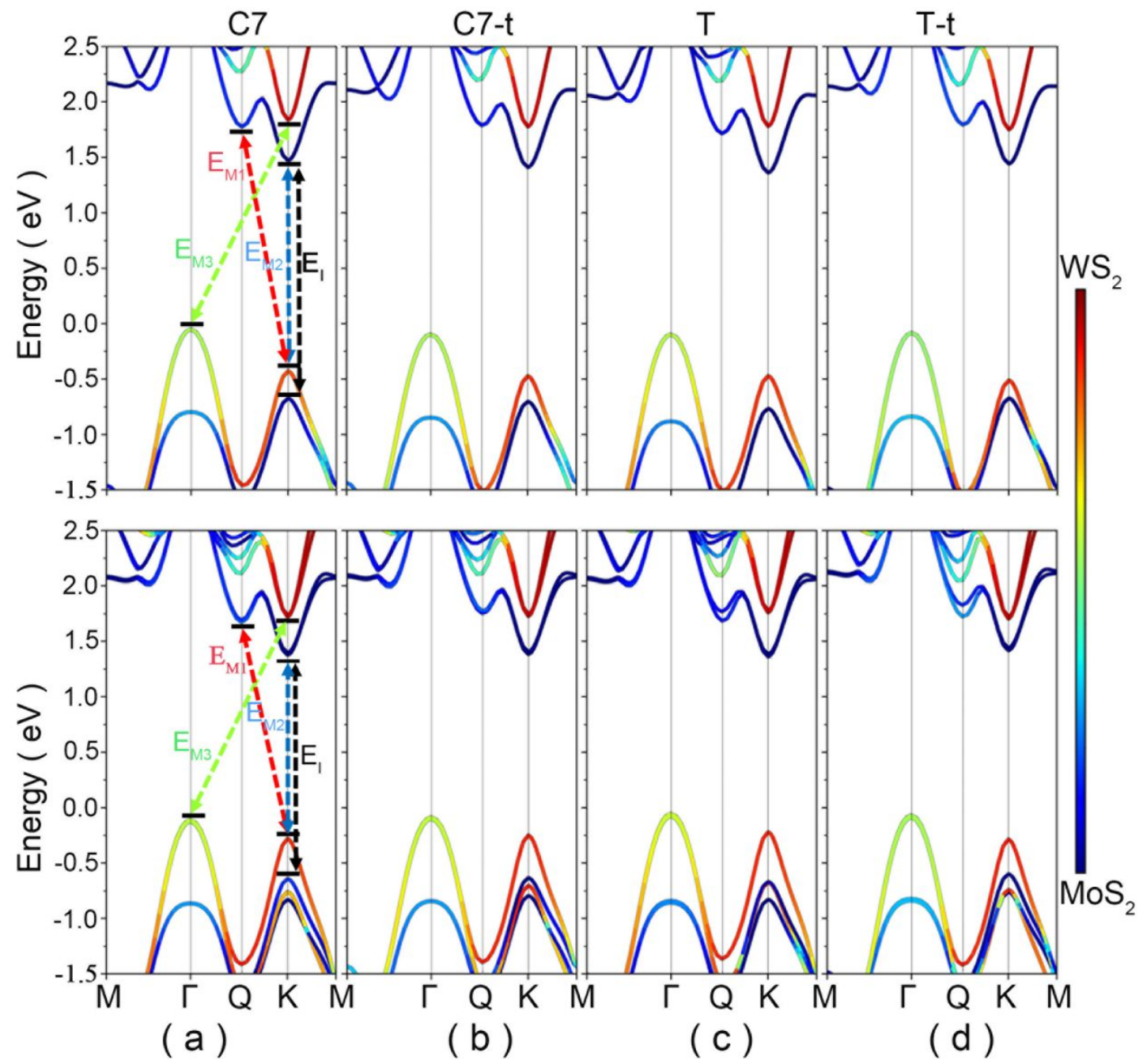

Figure S4. The band structures of $\mathrm{MoS}_{2} / \mathrm{WS}_{2}$ heterostructure in (a) C7, (b) C7-t, (c) $\mathrm{T}$ and (d) T-t stacking calculated on the basis of HSE06 functionals. The 
corresponding band structures calculated with HSE06+SOC are shown in the bottom panel, respectively.

Table S2. The calculated single-particle energies of $\mathrm{E}_{\mathrm{I}}, \mathrm{E}_{\mathrm{M} 1}, \mathrm{E}_{\mathrm{M} 2}$ and $\mathrm{E}_{\mathrm{M} 3} \mathrm{using}$ HSE06 and HSE06+SOC, respectively. The minimal energy difference between $\mathrm{E}_{\mathrm{I}}$ and $\mathrm{E}_{\mathrm{M} 1} / \mathrm{E}_{\mathrm{M} 2} / \mathrm{E}_{\mathrm{M} 3}$ is denoted as $\Delta\left(\mathrm{E}_{\mathrm{IM}}\right)$.

\begin{tabular}{|c|c|c|c|c|c|c|}
\hline & & $\mathrm{E}_{\mathrm{I}}$ & $\mathrm{E}_{\mathrm{M} 1}$ & $\mathrm{E}_{\mathrm{M} 2}$ & $\mathrm{E}_{\mathrm{M} 3}$ & $\Delta\left(\mathrm{E}_{\mathrm{IM}}\right)$ \\
\hline \multirow{3}{*}{$\mathrm{C} 7$} & $\mathrm{HSE06}$ & 2.14 & 2.24 & 1.9 & 1.89 & -0.1 \\
\cline { 2 - 7 } & HSE06+SOC & 2.04 & 1.97 & 1.68 & 1.83 & 0.07 \\
\hline \multirow{2}{*}{$\mathrm{C} 7-\mathrm{t}$} & HSE06 & 2.11 & 2.26 & 1.88 & 1.88 & -0.15 \\
\cline { 2 - 8 } $\mathrm{T}$ & HSE06+SOC & 2.01 & 2.02 & 1.63 & 1.82 & -0.01 \\
\cline { 2 - 8 } & HSE06+SOC & 2.05 & 1.98 & 1.60 & 1.82 & 0.07 \\
\hline \multirow{2}{*}{$\mathrm{T}-\mathrm{t}$} & HSE06 & 2.13 & 2.3 & 1.95 & 1.83 & -0.17 \\
\cline { 2 - 8 } & HSE06+SOC & 2.04 & 2.11 & 1.73 & 1.77 & -0.07 \\
\hline
\end{tabular}

\section{REFERENCES}

1. Gross, E. K. U.; Kohn, W., Local density-functional theory of frequency-dependent linear response. Phys. Rev. Lett. 1985, 55 (26), 2850.

2. Marques, M. A.; Maitra, N. T.; Nogueira, F. M.; Gross, E. K.; Rubio, A., Fundamentals of time-dependent density functional theory. Springer Science \& Business Media: 2012; Vol. 837.

3. Refaely-Abramson, S.; Jain, M.; Sharifzadeh, S.; Neaton, J. B.; Kronik, L., Solidstate optical absorption from optimally tuned time-dependent range-separated hybrid density functional theory. Phys. Rev. B 2015, 92 (8), 081204(R). 
4. Yanai, T.; Tew, D. P.; Handy, N. C., A new hybrid exchange-correlation functional using the Coulomb-attenuating method (CAM-B3LYP). Chem. Phys. Lett. 2004, 393 (1-3), 51-57.

5. Refaely-Abramson, S.; Sharifzadeh, S.; Jain, M.; Baer, R.; Neaton, J. B.; Kronik, L., Gap renormalization of molecular crystals from density-functional theory. Phys. Rev. B 2013, 88 (8), 081204(R).

6. Zhang, X.; Li, Z.; Lu, G., A non-self-consistent range-separated time-dependent density functional approach for large-scale simulations. J. Phys. Condens. Matter 2012, $24(20), 205801$.

7. Huang, L.-y.; Zhang, X.; Zhang, M.; Lu, G., Effect of point defects on optical properties of graphene fluoride: a first-principles study. J. Phys. Chem. C 2017, 121 (23), 12855-12862.

8. Casida, M. E., Time-dependent density functional response theory for molecules. In Recent Advances In Density Functional Methods: (Part I), World Scientific: 1995; pp 155-192.

9. Zhang, X.; Li, Z.; Lu, G., First-principles simulations of exciton diffusion in organic semiconductors. Phys. Rev. B 2011, 84 (23), 235208.

10. Torun, E.; Miranda, H. P.; Molina-Sánchez, A.; Wirtz, L., Interlayer and intralayer excitons in MoS 2/WS 2 and MoSe 2/WSe 2 heterobilayers. Phys. Rev. B 2018, 97 (24), 245427.

11. Qiu, D. Y.; Felipe, H.; Louie, S. G., Screening and many-body effects in twodimensional crystals: Monolayer MoS 2. Phys. Rev. B 2016, 93 (23), 235435.

12. Mak, K. F.; Lee, C.; Hone, J.; Shan, J.; Heinz, T. F., Atomically thin MoS 2: a new direct-gap semiconductor. Phys. Rev. Lett. 2010, 105 (13), 136805.

13. Splendiani, A.; Sun, L.; Zhang, Y.; Li, T.; Kim, J.; Chim, C.-Y.; Galli, G.; Wang, F., Emerging photoluminescence in monolayer MoS2. Nano Lett. 2010, 10 (4), 12711275.

14. Ye, Z.; Cao, T.; O’brien, K.; Zhu, H.; Yin, X.; Wang, Y.; Louie, S. G.; Zhang, X., Probing excitonic dark states in single-layer tungsten disulphide. Nature 2014, 513 (7517), 214-218. 
15. Hanbicki, A.; Currie, M.; Kioseoglou, G.; Friedman, A.; Jonker, B., Measurement of high exciton binding energy in the monolayer transition-metal dichalcogenides WS2 and WSe2. Solid State Commun. 2015, 203, 16-20.

16. Andersen, K.; Latini, S.; Thygesen, K. S., Dielectric genome of van der Waals heterostructures. Nano Lett. 2015, 15 (7), 4616-4621. 